\title{
Sorption of aromatic hydrocarbons onto montmorillonite as affected by norfloxacin
}

\author{
Zhiguo Pei ${ }^{\mathrm{a}, *}$, Jingjing Kong ${ }^{\mathrm{b}}$, Xiao-quan Shan $^{\mathrm{a}}$, Bei Wen ${ }^{\mathrm{a}}$ \\ a State Key Laboratory of Environmental Chemistry and Ecotoxicology, Research Center for Eco-Environmental Sciences, Chinese Academy of Sciences, PO Box 2871, Beijing 100085, \\ China

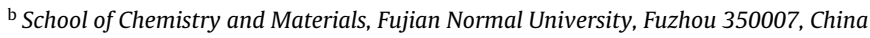

\section{A R T I C L E I N F O}

\section{Article history:}

Received 26 August 2011

Received in revised form

28 November 2011

Accepted 28 November 2011

Available online 8 December 2011

\section{Keywords:}

Sorption

Norfloxacin

Aromatic hydrocarbons

Montmorillonite

${ }^{1} \mathrm{H}$ NMR

\begin{abstract}
A B S T R A C T
Effect of norfloxacin (Nor) on the sorption of 1,3-dinitrobenzene (1,3-DNB), and PAHs (naphthalene (NAPH), phenanthrene (PHEN) and pyrene (PYR)) to $\mathrm{K}^{+}$-montmorillonite was studied. Nor suppressed 1,3-DNB sorption due to their competition for the same sorption sites. 1,3-DNB was sorbed on $\mathrm{K}^{+}-$ montmorillonite surface via cation-polar interaction and $n-\pi$ electron donor-acceptor interaction. Nor also was sorbed on these sites through cation exchange, cation bridging and/or surface complexation. Nor increased three PAHs sorption on montmorillonite and the enhanced magnitude was positively correlated with the $\pi$-donor strength of three PAHs. The enhanced sorption of PAHs by Nor was primarily attributed to $\pi-\pi$ interaction between $\pi$-electron-depleted quinoline ring of Nor and $\pi$-electron-rich PAHs. Compared with cation $\left(\mathrm{Nor}^{+}\right)$and anion $\left(\mathrm{Nor}^{-}\right)$, zwitterion $\left(\mathrm{Nor}^{ \pm}\right)$of Nor increased PHEN and PYR sorption more pronounced due to additional cation $-\pi$ interaction between the sorbed Nor $^{ \pm}$and PAHs. ${ }^{1} \mathrm{H}$ NMR spectrum provided direct evidence for $\pi-\pi$ and cation $-\pi$ complexation between PAHs and Nor $^{+}$ in solution by ring-current-induced upfield chemical shifts of amino group and methylene group of Nor ${ }^{+}$.
\end{abstract}

(c) 2011 Elsevier B.V. All rights reserved.

\section{Introduction}

Recently, antibiotics residue in the environment is becoming a growing concern. Many antibiotics can be accumulated to levels as high as $\mathrm{mg} \mathrm{kg}^{-1}$ in soils through land application of fertilizers, sewage sludge, wastewater irrigation or discard of outof-date pharmaceutical prescriptions [1]. Most of antibiotics have hydrophilic acidic and/or alkaline functional groups within their molecules, and can exist in aqueous solution as cation, zwitterion and/or anion. Thus, their sorption behavior in the environment is significantly different from that of other hydrophobic organic chemicals (HOCs). Past studies examined $\mathrm{pH}$-dependent sorption of antibiotics onto clays [2], aluminum oxides [3,4], and amorphous iron oxides [3], goethite [4], soil and soil minerals [5,6], and carbon nanotubes [7]. The proposed mechanisms included cation exchange and cation bridging [8], surface complexation [3,4], and hydrophobic interaction [7].

Nitroaromatic compounds (NACs) are a group of polar chemicals that are widely used as explosives, pesticides, solvents, and intermediates for organic synthesis. Contamination of NACs in soils and groundwater aquifers is common and has drawn considerable

\footnotetext{
* Corresponding author. Tel.: +86 10 62849329; fax: +861062923563.

E-mail address: peizg@rcees.ac.cn (Z. Pei).
}

attention $[8,9]$. It has been recognized that clay minerals, especially those exchanged with weakly hydrated cations $\left(\mathrm{K}^{+}, \mathrm{Cs}^{+}\right)$, can strongly retain NACs [10-12]. Two mechanisms have been proposed: (1) $n-\pi$ electron-donor-acceptor (EDA) interaction of NACs ( $\pi$-electron acceptors) with lone electron pairs of siloxane oxygens (n-donors) $[10,11]$ and (2) cation-polar interaction of nitro groups with exchangeable cations [12-14]. There is scientific debate on the concurrence and relative importance of these two mechanisms for sorption of NACs to clay minerals for a long time. Recently, Qu et al. compared the sorption of hexafluorobenzene and dinitrobenzenes on montmorillonite, and suggested that sorption of hexafluorobenzene was dominated by $n-\pi$ EDA interaction, while sorption of nitroaromatics was dominated by cation-polar interaction due to highly delocalized electrons (oxygen is partially negatively charged) on their nitro groups [15].

PAHs are a group of ubiquitous, nonionic HOCs due to their toxicity and suspected carcinogenity. PAHs sorption from water to solid phase is thought to be primarily due to their interaction with immobile organic matter [16]. However, for sorbents containing only very small amount of organic carbon, mineral phase was found to contribute significantly to the sorption of PAHs [17]. The sorption mechanisms of PAHs on clay minerals were controversial. Several models have been outlined to describe the driving forces for sorption of PAHs by mineral surfaces: (1) hydrophobic interaction, which was driven by a substantial thermodynamic gradient 
due to a combination of relatively small van der Waals forces and large entropy differences [18,19]; (2) partition from bulk aqueous phase to vicinal water film on montmorillonite surface $[20,21]$; (3) cation $-\pi$ interaction between the accumulated "soft" cations on clay mineral surface and aromatic ring [22]; (4) capillary condensation into interlayer nano or micropores of montmorillonite [17].

It is common that antibiotics, NACs and PAHs may coexist in the contaminated environment. However, most of the past sorption studies often focused on antibiotics, NACs or PAHs separately in order to explore their underlying sorption mechanisms onto clays more easily. To our knowledge, there are very few investigations on the influence of zwitterionic chemicals on the sorption of NACs or PAHs to clays. Although natural organic matter (NOM) and surfactants were also ionic organic chemicals, and could affect the sorption of other organic chemicals [23-25]. However, such influence was generally attributed to aliphatic chains in their molecules [26]. Thus, knowledge obtained from NOM and surfactants may not be applied to other zwitterionic chemicals.

In this study, we chose norfloxacin (Nor) as a common representative of zwitterionic organic chemicals, and examined its effects on the sorption of one NACs (1,3-DNB), and three PAHs (NAPH, PHEN and PYR). Nor has two $\mathrm{p} K_{\mathrm{a}}$ (6.22 and 8.51), and can exist either in $\mathrm{Nor}^{+}, \mathrm{Nor}^{ \pm}$or Nor ${ }^{-}$form in aqueous solution at different $\mathrm{pH}$ conditions due to the presence of two proton-binding sites (carboxyl and piperazinyl groups) (Fig. S1). The objectives of this study were (i) to reveal the dissociation influences of Nor on the sorption of aromatics on $\mathrm{K}^{+}$-montmorillonite and (ii) to provide an insight to the interactions between Nor and four aromatics during their sorption, which helps to understand the underlying mechanisms responsible for Nor effect.

\section{Materials and methods}

\subsection{Chemicals}

Analytical reagent grade of Nor, 1,3-DNB, NAPH, PHEN, and PYR were obtained from Sigma-Aldrich Co. (St. Louis, MO). The structures and properties of these organic chemicals are shown in Table S1.

\subsection{Sorbent}

Montmorillonite (purity $>98 \%$ ) was purchased from Beijing Youlichuangjia Science and Technology Development Company (Beijing, China). A fraction of $<2.0 \mu \mathrm{m}$ was obtained by wet sedimentation and subsequently exchanged with $\mathrm{K}^{+}$using chloride salts, dialyzed free of excess salt, and freeze-dried. The freeze-dried samples were stored in plastic bottles. The cation exchange capacity (CEC) of $\mathrm{K}^{+}$-montmorillonite is $107.1 \mathrm{cmol} \mathrm{kg}^{-1}$ by compulsive exchange with $\mathrm{Ba}^{2+}[27]$.

\subsection{Sorption experiments}

All sorption experiments were conducted in triplicates using a batch equilibration technique at $23 \pm 1^{\circ} \mathrm{C}$. For sorption isotherm experiments, the suspended solution $\mathrm{pH}$ was between 6.9 and 7.2 (measured at the end of batch sorption). Under this pH condition, Nor is zwitterion. For the $\mathrm{pH}$ effect experiments, the suspended solution $\mathrm{pH}$ was adjusted with a weighed amount of $\mathrm{HCl}$ or $\mathrm{KOH}$ solutions. The final equilibrium $\mathrm{pH}$ was measured at the end of batch experiments.

For sorption of 1,3-DNB (1.0, 3.0, 6.0, 10.0, 14.0, and $20.0 \mathrm{mg} / \mathrm{L})$, NAPH $(0.1,0.5,1.0,2.0,3.0$, and $5.0 \mathrm{mg} / \mathrm{L})$, PHEN $(0.1,0.2,0.3$, $0.5,0.7$ and $1.0 \mathrm{mg} / \mathrm{L})$, and PYR $(0.01,0.02,0.03,0.05,0.07$ and
$0.1 \mathrm{mg} / \mathrm{L})$ in the presence of Nor $(0,20$, and $50 \mathrm{mg} / \mathrm{L})$, the sorption experiments were carried out by mixing a certain amount of $\mathrm{K}^{+}$-montmorillonite with corresponding $0.01 \mathrm{M} \mathrm{KCl}$ solution in $50 \mathrm{~mL}$ glass centrifuge tubes sealed with Teflon-lined screw caps. Sorbates in methanol were added using a microsyringe, and the volume percentage of methanol was kept below $0.1 \%$ to minimize cosolvent effects. The prepared mixed samples were vortexed for $10 \mathrm{~s}$ and agitated on a flat bed shaker for $24 \mathrm{~h}$ at $23 \pm 1^{\circ} \mathrm{C}$ (apparent equilibrium was reached before this time). Controls (without any $\mathrm{K}^{+}$-montmorillonite) were also established. Results showed that no significant loss of sorbates was observed (recoveries always $>97 \%$ ), indicating that sorption to glassware and degradation of these chemicals were negligible (data not shown). The suspension was centrifuged at $1667 \times g$ for $20 \mathrm{~min}$ to separate liquid from solid phases, and the concentrations of the solutes in the supernatant phase were determined by HPLC.

The $\mathrm{pH}$ effect experiments were conducted using a batch sorption approach as mentioned above. The initial concentrations of four sorbates were 0 and $50 \mathrm{mg} / \mathrm{L}$ Nor, $5.0 \mathrm{mg} / \mathrm{L}$ 1,3-DNB, $1.0 \mathrm{mg} / \mathrm{L}$ NAPH and PHEN, and $0.1 \mathrm{mg} / \mathrm{L}$ PYR, respectively. After equilibrium was reached, the suspension was centrifuged, and the sorbate concentrations in the supernatants were determined by HPLC. The sorbed mass was calculated from the differences between the initial and final equilibrium concentrations.

\subsection{Attenuated total reflectance Fourier transform infrared (ATR-FTIR) spectroscopic measurement}

ATR-FTIR spectra were obtained on a Nicolet Nexus 470 FTIR spectrometer equipped with a ZnSe crystal fitted in a horizontal attenuated total reflectance (HATR) cell (Pike Technologies). A total of 256 scans with a spectral resolution of $4 \mathrm{~cm}^{-1}$ were employed.

Samples of Nor for ATR-FTIR analyses were prepared at $\mathrm{pH}$ $4.5 \pm 0.1,7.0 \pm 0.1$, and $9.0 \pm 0.1$, respectively. At $\mathrm{pH} 4.5,7.0$ or 9.0 , the dominant species of Nor were cation $\left(\mathrm{Nor}^{+}\right)$, zwitterion $\left(\mathrm{Nor}^{ \pm}\right)$ and anion (Nor ${ }^{-}$) in solution, respectively. After sorption and centrifugation, the paste and supernatant were collected, separately, and immediately spread on the ZnSe crystal surface to obtain an appropriate thin layer. The sample holding region was sealed with a lid to prevent evaporation, and FTIR spectra were measured. FTIR spectra of $\mathrm{K}^{+}$-montmorillonite in $0.01 \mathrm{M} \mathrm{KCl}$ were also measured in the absence of Nor at three defined pHs. For aqueous solution of Nor, difference spectra at each $\mathrm{pH}$ were obtained by subtracting the spectra of background electrolyte solution from the spectra of Nor solutions. For the sorbed Nor on $\mathrm{K}^{+}$-montmorillonite, the difference spectra were obtained by subtracting the spectra of $\mathrm{K}^{+}$montmorillonite from corresponding spectra of the sorbed Nor on $\mathrm{K}^{+}$-montmorillonite.

\subsection{Solution-phase ${ }^{1} H$ NMR}

${ }^{1} \mathrm{H}$ NMR spectra were recorded at room temperature using a Bruker-DRX $500 \mathrm{MHz}$ spectrometer for cationic Nor in mixtures with model $\pi$-donor compounds (NAPH, PHEN and PYR) in dimethylsulfoxide- $d_{6}$ (99.8\% deuterium).

\subsection{Aqueous solubility enhancement}

Aqueous solubility of PAHs (NAPH, PHEN, and PYR) was measured in the Nor ${ }^{+}$and Nor $^{-}$solution, respectively. Vials containing an organic compound and $20 \mathrm{~mL}$ different concentrations of $\mathrm{Nor}^{+}$ or Nor ${ }^{-}$solution were shaken in an orbital shaker at $23 \pm 1^{\circ} \mathrm{C}$ for at least $5 \mathrm{~d}$. After centrifugation, an aliquot of aqueous phase was withdrawn carefully for analysis of solute concentration. 


\subsection{Analysis}

Solutes in the supernatants were determined by an Agilent 1200 reversed-phase HPLC (Atlantis-dC18 ODS HPLC column, $4.6 \mathrm{~mm}$ i.d. $\times 150 \mathrm{~mm}, 5 \mu \mathrm{m}$ ) equipped with UV and fluorescence detectors. The determination wavelengths were set at $262 \mathrm{~nm}$ for 1,3-DNB. The fluorescence wavelengths for measurement of Nor, NAPH, PHEN, and PYR were at the excitation wavelengths of 280, 225, 250 and $275 \mathrm{~nm}$, and at the emission wavelengths of 450, 335, 364 and $394 \mathrm{~nm}$, respectively. The mobile phase for Nor is acetonitrile:water (pH 3.0, adjusted with formic acid) $(20: 80, \mathrm{v}: \mathrm{v})$ at a flow rate of $0.8 \mathrm{~mL} \mathrm{~min}^{-1}$. Acetonitrile and water mixture at a volume ratio of $90 \%$ to $70 \%$ was used at a flow rate of $1.0 \mathrm{~mL} \mathrm{~min}^{-1}$ as the mobile phase for the determination of NAPH, PHEN and PYR.

\subsection{Data analysis}

Freundlich equation can be expressed as $Q=K_{\mathrm{f}} C^{n}$, where $Q$ $(\mathrm{mg} / \mathrm{g})$ is the amount of sorbed organic compounds by $\mathrm{K}^{+}-$ montmorillonite, $C(\mathrm{mg} / \mathrm{L})$ is the equilibrium concentration in solution, $K_{\mathrm{f}}$ is an empirical constant related to sorption capacity $\left(\mathrm{mg}^{(1-n)} \mathrm{g}^{-1} \mathrm{~mL}^{n}\right)$, and $n$ is the Freundlich exponent or a site energy heterogeneity factor, often used as an indicator of isotherm nonlinearity.

\section{Results and discussion}

\subsection{Influence of solution $\mathrm{pH}$ on Nor sorption}

It was found that Nor sorption to $\mathrm{K}^{+}$-montmorillonite was almost $\mathrm{pH}$-independent between $\mathrm{pH} \sim 4.0-8.0$ (Fig. S2). When $\mathrm{pH}$ was $>\sim 8.0$ Nor sorption to $\mathrm{K}^{+}$-montmorillonite was remarkably decreased due to repulsion between $\mathrm{Nor}^{-}$and negatively charged surface of $\mathrm{K}^{+}$-montmorillonite (Fig. S3). To examine the contributions of individual Nor species to overall sorption, we calculated species-specific equilibrium sorption contribution [28]. The overall sorbed amount at a given $\mathrm{pH}$ value can be represented as the sum of the contributions of individual species:

$C s=C s^{\mathrm{Nor}+} a_{\mathrm{Nor}+}+C s^{\mathrm{Nor} \pm} \cdot a_{\mathrm{Nor} \pm}+C s^{\mathrm{Nor}-} \cdot a_{\mathrm{Nor}-}$

where $C s(\mathrm{mg} / \mathrm{g})$ is the overall equilibrium sorption concentrations; $\mathrm{Cs}^{\mathrm{Nor}+}, \mathrm{Cs}^{\mathrm{Nor} \pm}$ and $\mathrm{Cs}^{\mathrm{Nor}-}$ are the sorption contribution of cationic, zwitterionic and anionic species, respectively; and $\alpha_{\mathrm{Nor}^{+}}, \alpha_{\mathrm{Nor} \pm}$ and $\alpha_{\text {Nor- }}$ represent the mass fractions of these species in bulk solution. The best fittings were obtained for $\mathrm{Cs}^{\mathrm{Nor}+}$ of $41.0 \pm 0.7 \mathrm{mg} / \mathrm{g}$, $C s^{\text {Nor }} \pm$ of $39.2 \pm 0.5 \mathrm{mg} / \mathrm{g}$ and $C \mathrm{~S}^{\mathrm{Nor}-}$ of $26.4 \pm 0.9 \mathrm{mg} / \mathrm{g}$ at the initial concentration of Nor $50.0 \mathrm{mg} / \mathrm{L}$ (Table S2).

ATR-FTIR was used to study the sorption mechanisms of Nor at different pHs. Fig. 1a-c shows FTIR spectra of Nor in aqueous solution at different pHs. At $\mathrm{pH} 4.5$, a peak at $\sim 1745 \mathrm{~cm}^{-1}$ appeared, corresponding to $\mathrm{C}=0$ stretch $\left(v_{\mathrm{C}=0 \text { carboxyl }}\right)$ of $-\mathrm{COOH}$ group. When $\mathrm{pH}$ was increased to 7.0 and 9.0 , the intensity of $v_{\mathrm{C}=\mathrm{O} \text { carboxyl }}$ decreased, concurrently, the asymmetric $\left(\sim 1582 \mathrm{~cm}^{-1}\right)$ and symmetric $\left(\sim 1341 \mathrm{~cm}^{-1}\right)$ stretch of $\mathrm{COO}^{-}$ appeared due to deprotonation of $-\mathrm{COOH}$ group. Assignments of the remaining vibrations observed in Fig. 1 are as follows: $\mathrm{C}=\mathrm{O}$ stretch of ketone group $\left(1629 \mathrm{~cm}^{-1}\right)$, and coupled carboxylic acid $\mathrm{C}-\mathrm{O}$ stretch $\left(v_{\mathrm{COOH}}\right)$ and $\mathrm{O}-\mathrm{H}$ deformation $\left(\delta_{\mathrm{C}-\mathrm{OH}}\right)\left(1274 \mathrm{~cm}^{-1}\right)$ $[3,4]$. The interaction between Nor and $\mathrm{K}^{+}$-montmorillonite was investigated by comparing the spectra of sorbed Nor with those of Nor in solution. When Nor was sorbed on $\mathrm{K}^{+}$-montmorillonite (Fig. 1d) at $\mathrm{pH} 4.5$, the spectrum of sorbed Nor $^{+}$was similar to that of $\mathrm{Nor}^{+}$in aqueous solution, where the peaks of carboxyl group $\left(1744 \mathrm{~cm}^{-1}\right)$ and keto group $\left(1629 \mathrm{~cm}^{-1}\right)$ exhibited no shift. Because outer-sphere complexes and aqueous $\mathrm{Nor}^{+}$species had similar molecular geometries [29], outer-sphere complex was

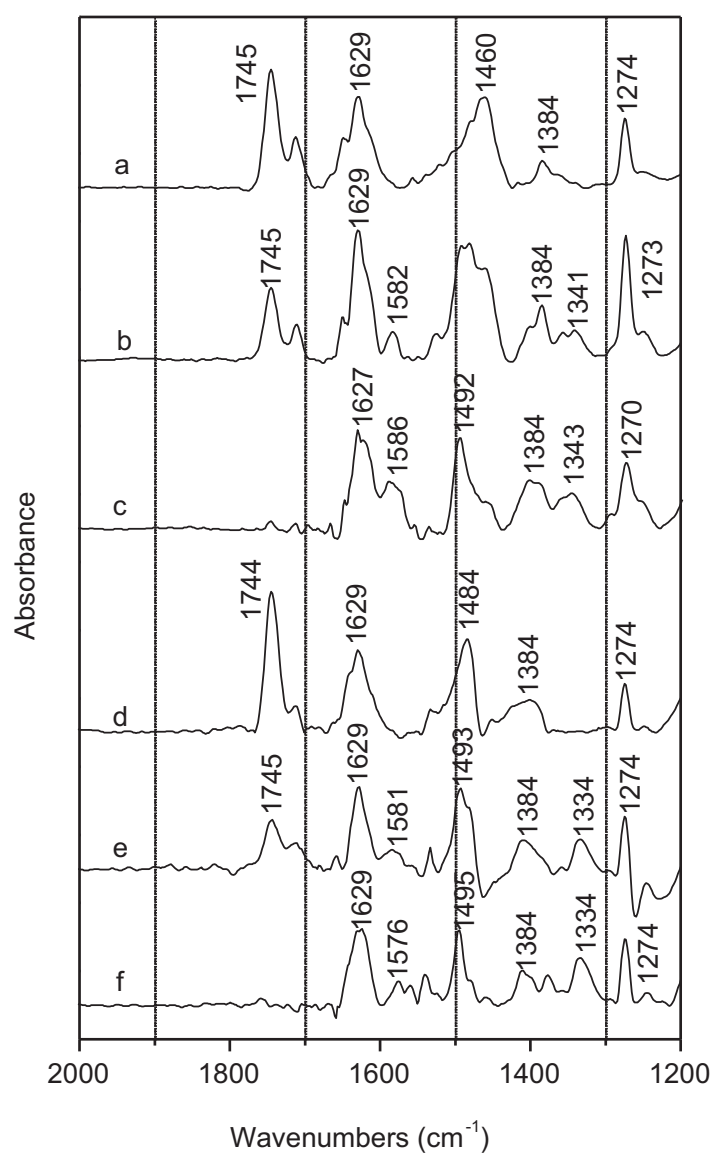

Fig. 1. FTIR spectra of Nor in solution at pH 4.5 (a), 7.0 (b), 9.0 (c) and on $\mathrm{K}^{+}-$ montmorillonite at pH 4.5 (d), 7.0 (e), and 9.0 (f).

expected to form for $\mathrm{Nor}^{+}$on montmorillonite surface through electrostatic interaction between negatively charged sites on mineral and protonated heterocyclic $\mathrm{N}$ atom of $\mathrm{Nor}^{+}$. At $\mathrm{pH} 7.0$, the absorbance of $\mathrm{COO}^{-}$symmetric stretch of $\mathrm{Nor}^{ \pm}$shifted from 1341 to $1334 \mathrm{~cm}^{-1}$, indicating that at least a portion of $\mathrm{Nor}^{ \pm}$was sorbed on $\mathrm{K}^{+}$-montmorillonite surface through interaction of its carboxyl group. In this case, we could not also rule out sorption of other portion of Nor ${ }^{ \pm}$on montmorillonite surface through electrostatic interaction via its protonated heterocyclic $\mathrm{N}$ atom [6]. If $\mathrm{pH}$ was further increased to 9.0, the asymmetric and symmetric stretch of $\mathrm{COO}^{-}$shifted from 1586 and $1343 \mathrm{~cm}^{-1}$ to 1576 and $1334 \mathrm{~cm}^{-1}$, respectively (Fig. 1f), indicating that carboxyl group of Nor was responsible for the sorbed $\mathrm{Nor}^{-}$on $\mathrm{K}^{+}$-montmorillonite at $\mathrm{pH} 9.0$ [2].

Based on the above evidences from batch sorption experiments and FTIR study it may be surmised that multiple mechanisms are expected to operate simultaneously for the sorption of Nor onto $\mathrm{K}^{+}$-montmorillonite at different $\mathrm{pH}$ : (i) cation exchange occurred via columbic attraction of protonated heterocyclic $\mathrm{N}$ atom of $\mathrm{Nor}^{+}$ to negatively charged surface sites on $\mathrm{K}^{+}$-montmorillonite and via ion exchange with naturally occurring cations associated with sites of permanent negative charge on $\mathrm{K}^{+}$-montmorillonite or $\mathrm{pH}$ dependent negative charge arising from deprotonation of surface hydroxyl groups. Because in these reactions the sorption sites were not limited by the availability of cation exchange sites of $\mathrm{K}^{+}$montmorillonite but limited by Nor $^{+}$and Nor $^{ \pm}$, therefore, cation exchange appears to be the main mechanism for the sorption of Nor $^{+}$at $\mathrm{pH} 4.5$ and $\mathrm{Nor}^{ \pm}$at $\mathrm{pH}$ 7.0. (ii) Cation bridging was thought to occur via interaction between $\mathrm{COO}$ - group and exchangeable cations of negatively charged sites of $\mathrm{K}^{+}$-montmorillonite at $\mathrm{pHs} 7.0$ 
Table 1

Freundlich constants ( $K_{\mathrm{f}}$ and $n$ ) and correlation coefficients $(r)$ of 1,3-DNB, NAPH, PHEN, and PYR sorption onto montmorillonite $(n=3)$.

\begin{tabular}{lcllll}
\hline Sorbates & Nor $(\mathrm{mg} / \mathrm{L})$ & $K_{\mathrm{f}}$ & $n$ & $r$ & $K_{\mathrm{d}}(\mathrm{L} / \mathrm{g})$ \\
\hline 1,3-DNB & 0 & 0.333 & 0.70 & 0.97 & 0.23 \\
& 20 & 0.122 & 0.86 & 0.98 & 0.10 \\
& 50 & 0.07 & 0.89 & 0.99 & 0.06 \\
NAPH & 0 & 0.037 & 0.88 & 0.97 & 0.05 \\
& 20 & 0.043 & 0.84 & 0.98 & 0.07 \\
& 50 & 0.048 & 0.83 & 0.99 & 0.14 \\
PHEN & 0 & 0.022 & 0.67 & 0.99 & 0.08 \\
& 20 & 0.094 & 0.74 & 0.99 & 0.17 \\
& 50 & 0.294 & 0.85 & 0.99 & 0.42 \\
PYR & 0 & 0.204 & 0.79 & 0.98 & 0.36 \\
& 20 & 0.609 & 0.84 & 0.99 & 1.07 \\
& 50 & 0.626 & 0.83 & 0.98 & 4.31 \\
\hline
\end{tabular}

and 9.0. (iii) Surface complexation seemed plausible via the binding of carboxyl group to surficial aluminum on the edge sites of aluminosilicate at pHs 7.0 and 9.0. Currently, it is difficult to define to what extent each mechanism contributes to the overall sorption.

\subsection{Effect of Nor on the sorption of 1,3-DNB, NAPH, PHEN and PYR}

Fig. 2 shows the sorption isotherms of 1,3-DNB, NAPH, PHEN, and PYR on $\mathrm{K}^{+}$-montmorillonite in the presence and absence of Nor. These isotherms fitted Freundlich equation well, and were clearly nonlinear as characterized by low $n$ values (Table 1 ).

As shown in Fig. 2a, the sorption of $1,3-\mathrm{DNB}$ on $\mathrm{K}^{+}-$ montmorillonite decreased with increasing Nor concentrations from 0 to $50 \mathrm{mg} / \mathrm{L}$, suggesting that Nor suppressed the sorption of 1,3-DNB. For example, the sorption distribution coefficients $\left(K_{\mathrm{d}}\right)$ (initial concentration of $5.0 \mathrm{mg} / \mathrm{L}$ ) were used to compare the sorption behavior of 1,3-DNB (Table 1 ). Obviously, the $K_{\mathrm{d}}$ values decreased from 0.23 to $0.06 \mathrm{~L} / \mathrm{g}$ when Nor concentrations increased from 0 to $50 \mathrm{mg} / \mathrm{L}$. Nonlinear sorption of 1,3-DNB suggested the presence of specific interactions between 1,3-DNB and $\mathrm{K}^{+}$-montmorillonite. Fig. S4 shows the FTIR spectra of sorbed 1,3DNB on $\mathrm{K}^{+}$-montmorillonite, where a red shift of $v_{\text {asym }}(\mathrm{NO})$ band of 1,3-DNB from $1542 \mathrm{~cm}^{-1}$ to $1536 \mathrm{~cm}^{-1}$ and a concomitant blue shift of $v_{\text {sym }}$ (NO) band from $1350 \mathrm{~cm}^{-1}$ to $1356 \mathrm{~cm}^{-1}$ were found. This suggested that 1,3-DNB was sorbed on $\mathrm{K}^{+}$-montmorillonite through complexation of its $-\mathrm{NO}_{2}$ groups with exchangeable $\mathrm{K}^{+}$ cations of montmorillonite via cation-polar interaction and/or complexation between 1,3-DNB ( $\mathrm{e}^{-}$acceptors) and oxygens on siloxane surfaces of $\mathrm{K}^{+}$-montmorillonite ( $\mathrm{e}^{-}$donors) via $\mathrm{n}-\pi$ electron donor-acceptor (EDA) interaction $[11,13]$. When Nor was added to solution together, Nor was sorbed on montmorillonite through electrostatic interaction or cation bridging, which shielded sorption sites of 1,3-DNB on mineral surface and suppressed its sorption accordingly.

Sorption isotherms of NAPH, PHEN and PYR on $\mathrm{K}^{+}-$ montmorillonite in the presence and absence of Nor are shown in Fig. $2 \mathrm{~b}-\mathrm{d}$. For a given concentration, PYR had the highest $K_{\mathrm{d}}$ value on $\mathrm{K}^{+}$-montmorillonite among three PAHs, followed by PHEN and NAPH (Table 1). The order is consistent to hydrophobicity of three PAHs [5]. Thus, for three examined PAHs, hydrophobic interaction should be a main cause for their sorption on montmorillonite [19]. When Nor/NAPH, Nor/PHEN, or Nor/PYR combination system was sorbed on $\mathrm{K}^{+}$-montmorillonite (Fig. 2), the sorption of three PAHs increased with increasing Nor concentrations. The $K_{\mathrm{d}}$ values (initial concentration of $0.1 \mathrm{mg} / \mathrm{L}$ ) were used to compare the sorption behavior of three PAHs (Table 1 ). Obviously, the $K_{\mathrm{d}}$ values increased from 0.05 to $0.14 \mathrm{~L} / \mathrm{g}$ for NAPH, from 0.08 to $0.42 \mathrm{~L} / \mathrm{g}$ for PHEN, and from 0.36 to $4.2 \mathrm{~L} / \mathrm{g}$ for PYR when Nor concentrations increased from 0 to $50 \mathrm{mg} / \mathrm{L}$. Possible reason for the enhanced effect of Nor was that except for original PAHs sorption sites, the sorbed Nor formed new organic phase, thus created new sorption sites on $\mathrm{K}^{+}$-montmorillonite surface, and facilitated PAHs sorption [19]. As shown in Fig. 2, the enhanced magnitude of three PAHs sorption by Nor was strongly positive correlated with $\pi$-donor strength and $K_{\text {ow }}$ values of three PAHs (PYR $>$ PHEN $>$ NAPH). Therefore, it was proposed that main mechanisms about the enhancement effect of Nor on PAHs sorption likely resulted from $\pi-\pi$ interaction between electron-depleted quinoline ring ( $\pi$-electron acceptor) of Nor and PAHs ( $\pi$-electron donor) and/or hydrophobic interaction between the newly formed organic phase by Nor and PAHs.

To further evaluate the role of $\pi-\pi$ interaction on the overall enhancement effect, we chose pentachlorobenzene (PtCB) as a new sorbate, which has close hydrophobicity to PYR but has very less electron density due to electron induction effect of five chlorine atoms (Table S1). This means that there was similar hydrophobic interaction, but weaker $\pi-\pi$ interaction between PtCB and Nor compared to that between PYR and Nor [25]. If Nor could not increased the sorption of PtCB as much as PYR, it implied that the enhanced sorption of PAHs could be ascribed to $\pi-\pi$ interaction between Nor and PAHs [25]. As shown in Fig. S5, in the absence of Nor, PtCB and PYR had similar $K_{\mathrm{d}}$ values on $\mathrm{K}^{+}$montmorillonite. When Nor was added to solution, the sorption $K_{\mathrm{d}}$ values of PYR increased dramatically, however, the sorption $K_{\mathrm{d}}$ of PtCB only increased marginally. These results suggested that the enhanced sorption of PAHs by Nor was mainly due to $\pi-\pi$ interaction between Nor and PAHs rather than hydrophobic interaction.

The concentrations of Nor in the supernatants were also determined simultaneously (data not shown). Nor sorption on montmorillonite varied slightly in the presence of four organic solutes. Obviously, Nor sorption was independent of the presence of 1,3-DNB, NAPH, PHEN, and PYR. This phenomenon indirectly verified that Nor was preferentially sorbed on surface of $\mathrm{K}^{+}$-montmorillonite first through multiple mechanisms, and then PAHs were reacted with the sorbed Nor. It is plausible to assume that formation of ternary complex of $\mathrm{K}^{+}$montmorillonite-Nor-PAHs was likely.

\subsection{Effect of different species of Nor on the sorption of 1,3-DNB, NAPH, PHEN and PYR}

As described above, different species of Nor had different sorption mechanisms. And one wanted to know if different species of Nor had different effects on PAHs sorption. Fig. 3 shows the sorption of 1,3-DNB, NAPH, PHEN and PYR as a function of solution $\mathrm{pH}$ in the presence and absence of Nor. In the single solute system, it was found that the sorption of four adsorbates was almost independent of solution $\mathrm{pH}$ over the investigated $\mathrm{pH}$ range because these chemicals were nonionizable. When Nor was added to solution, it is found that solution $\mathrm{pH}$ had small effect on the sorption of 1,3-DNB on $\mathrm{K}^{+}$-montmorillonite. However, for PAHs, their sorption on $\mathrm{K}^{+}$montmorillonite was changed with solution pH. As shown in Fig. 3, solution $\mathrm{pH}$ had little effect on the sorption of NAPH, while for PHEN and PYR, their sorption on $\mathrm{K}^{+}$-montmorillonite increased significantly from $\mathrm{pH} 4.0$ to 7.0 , followed by a maximum at $\mathrm{pH} \sim 7.1$ and then decreased rapidly from $\mathrm{pH} 7.1$ to 10.0 . The increased sorption extent was consistent with $\pi$-donor strength of PAHs, indicating that delocalized $\pi$ electrons of PAHs likely played an important role in the overall enhancement effect. It should be noted that Nor sorption on $\mathrm{K}^{+}$-montmorillonite was also $\mathrm{pH}$ dependent at pH 7.0-10.0 (Fig. S2). To eliminate the effect of different sorption amount of Nor in different $\mathrm{pH}$ conditions, the $K_{\mathrm{d}}$ values of PHEN and PYR were normalized for the sorbed concentrations of Nor. As shown in Fig. S6, 

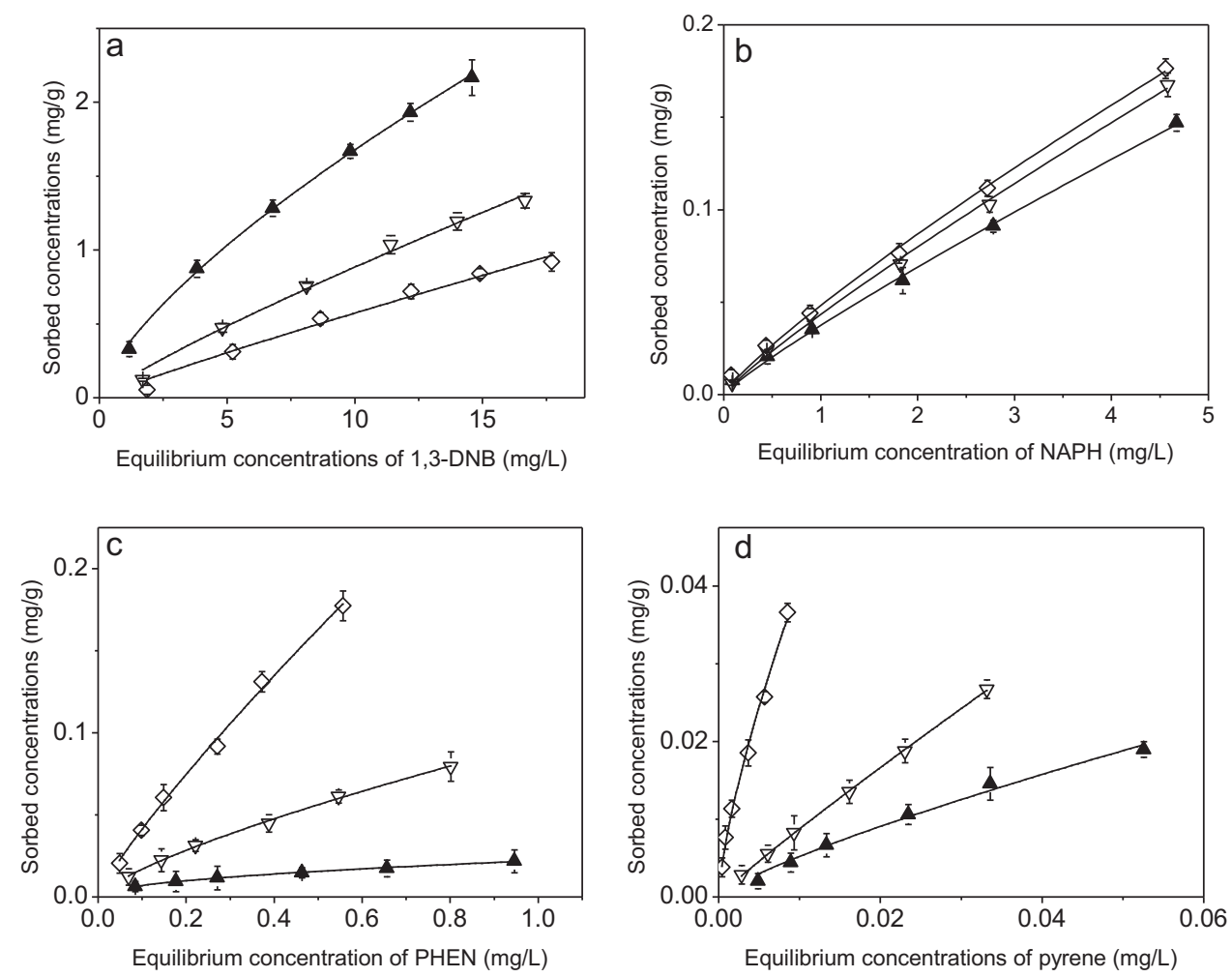

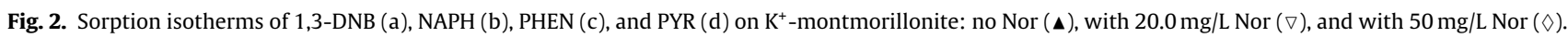

the sorption trend of PHEN and PYR as a function of $\mathrm{pH}$ has not been changed significantly. It was suggested that the effects of Nor on the sorption of PHEN and PYR was not only related to the sorbed amount of Nor, but also to different sorption mechanisms of $\mathrm{Nor}^{+}$, Nor $^{ \pm}$and Nor ${ }^{-}$on $\mathrm{K}^{+}$-montmorillonite.
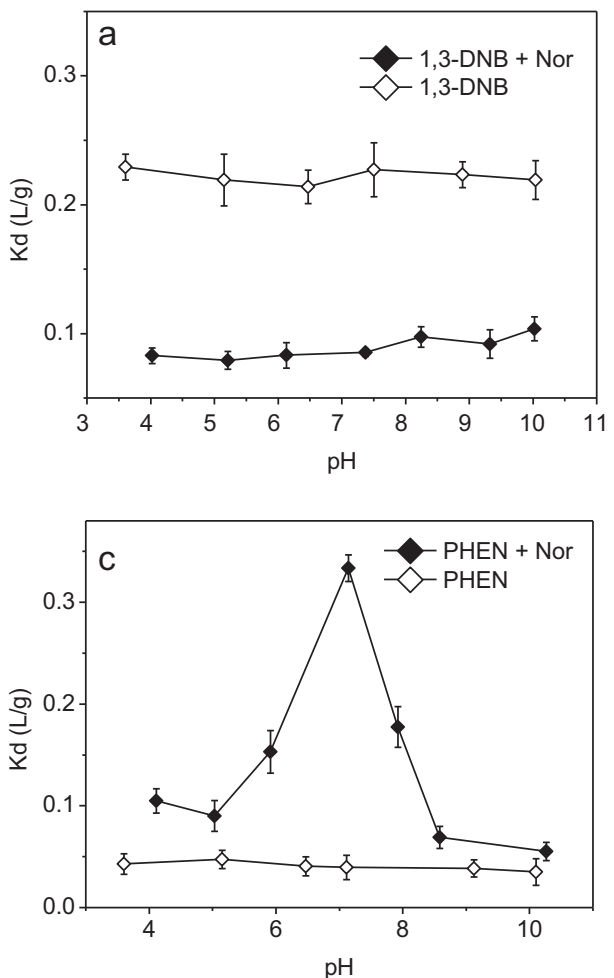

As described above, $\mathrm{Nor}^{+}$was sorbed on $\mathrm{K}^{+}$-montmorillonite through electrostatic interaction between protonated heterocyclic $\mathrm{N}$ atom of $\mathrm{Nor}^{+}$and negatively charged $\mathrm{K}^{+}$-montmorillonite surface, leaving electron-depleted quinoline ring ( $\pi$-electron acceptor) and $-\mathrm{COOH}$ group available freely. Several possible mechanisms
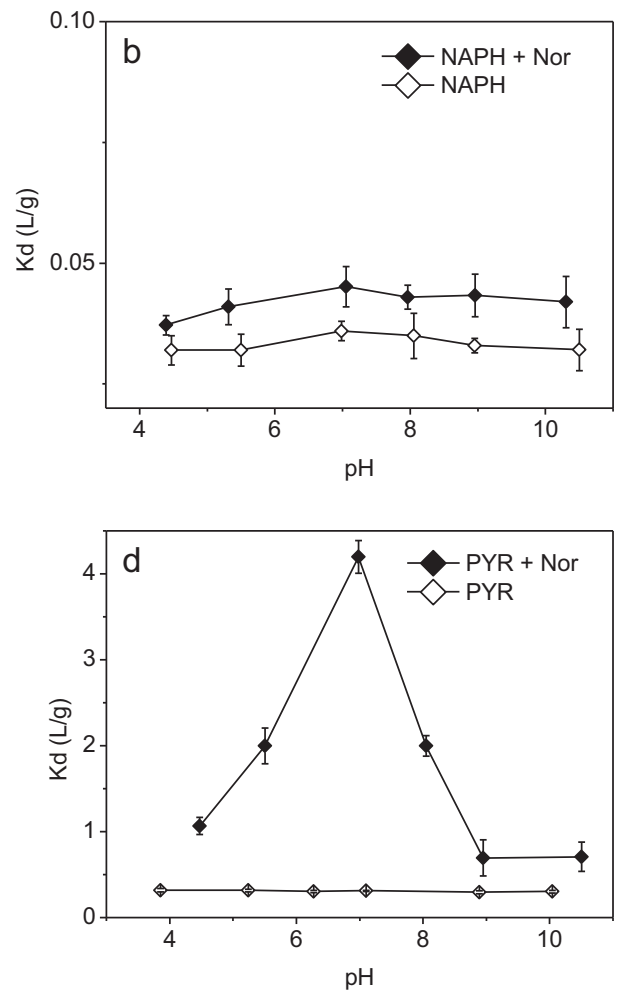

Fig. 3. Effect of equilibrium solution pH on the sorption of 1,3-DNB (a), NAPH (b), PHEN (c) and PYR (d) in the absence and presence of Nor on $\mathrm{K}^{+}$-montmorillonite. 

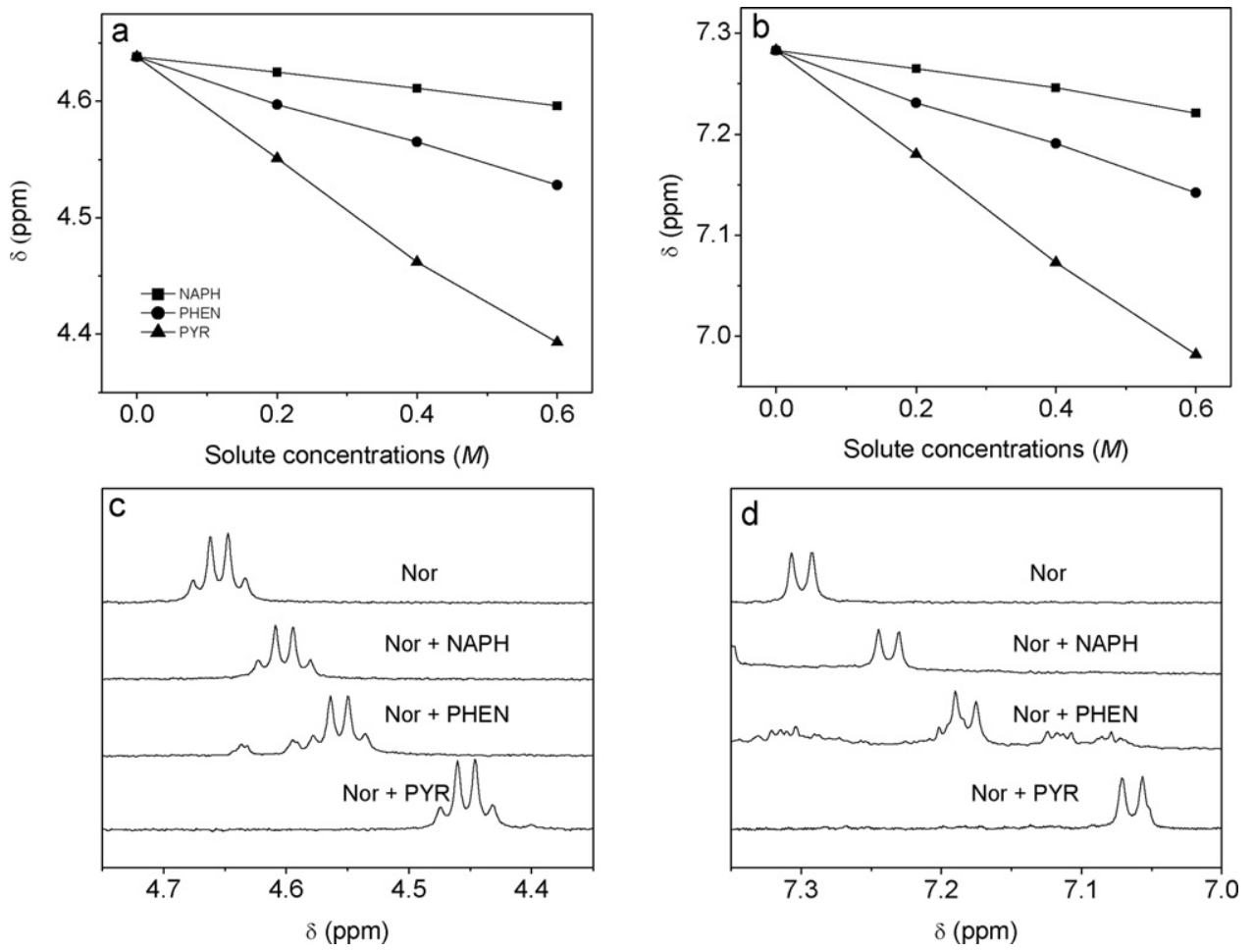

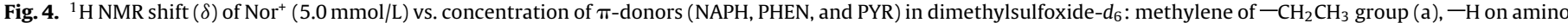
group of Nor (b). Selected NMR patterns of methylene of $-\mathrm{CH}_{2} \mathrm{CH}_{3}$ group (c), and $-\mathrm{H}$ on amino group of Nor (d).

should be considered based on the literature [30]: (1) $\pi-\pi$ electron donor-acceptor (EDA) interaction between electron-depleted quinoline ring ( $\pi$-electron acceptor) and PAHs ( $\pi$-electron donor); (2) hydrogen $-\pi$ bonding between $-\mathrm{COOH}$ group of sorbed $\mathrm{Nor}^{+}$ and aromatic rings of PHEN or PYR. Hydrogen $-\pi$ bonding was not likely a primary cause of the enhanced sorption for the following reason. When solution $\mathrm{pH}$ exceeds its $\mathrm{pKa}_{1}(6.22)$, $-\mathrm{COOH}$ group on Nor would be ionized, and hydrogen- $\pi$ bonding effect should have been significantly impeded. Fig. 3 shows that the sorption affinity did not decrease accordingly, while increased. At $\mathrm{pH} \sim 7.0$, a portion of $\mathrm{Nor}^{ \pm}$was sorbed on $\mathrm{K}^{+}$montmorillonite surface through interaction of its carboxyl group (Fig. 1). Thus, positively charged heterocyclic $\mathrm{N}$ atom of $\mathrm{Nor}^{ \pm}$was far away from mineral surface and freely available to PAHs ( $\pi$ electron donor). Except for $\pi-\pi$ interaction as described above, cation $-\pi$ should be another important reaction responsible for the enhanced sorption of PAHs by Nor ${ }^{ \pm}$. It was also the reason why the $K_{\mathrm{d}}$ values of PAHs in the presence of Nor $^{ \pm}$was remarkably increased compared to that in the presence of $\mathrm{Nor}^{+}$(Fig. 3). Cation $-\pi$ bonding between electron-rich aromatic structures and metal ions and ammonium ions toward molecular recognition and selective metal binding had been well documented in the literatures [15,31]. For example, $\mathrm{Qu}$ et al. found that the presence of tetra-alkyl ammonium largely enhanced the sorption NAPH, PHEN and PYR on smectite mainly due to formation of strong cation $-\pi$ EDA complexes between ammonium cation and PAHs [15]. For comparison, 1,3-DNB had an electron-depleted benzene ring, which was unfavorable for formation of cation- $\pi$ interaction with Nor ${ }^{ \pm}$. Similarly, Nor ${ }^{-}$was sorbed on $\mathrm{K}^{+}$-montmorillonite through the interaction of its carboxyl group (Fig. 1). The positive charge on heterocyclic $\mathrm{N}$ atom of $\mathrm{Nor}^{-}$and cation $-\pi$ interaction with PAHs disappeared. $\pi-\pi$ interaction should be responsible for the enhanced sorption of PAHs by Nor $^{-}$[30]. Compared to Nor $^{ \pm}$, PAHs sorption decreased rapidly at higher $\mathrm{pH}$ range (Fig. 3).

\section{4. ${ }^{1}$ H NMR studies}

The interactions between PAHs ( $\pi$-electron donors) and $\mathrm{Nor}^{+}$ ( $\pi$-electron acceptor) are supported by solution-phase ${ }^{1} \mathrm{H}$ NMR experiments. Placing a nucleus above or below an aromatic structure causes electronic shielding of the nucleus due to "ring current" effect. Thus, the ring current-induced upfield chemical shifts of ${ }^{1} \mathrm{H}$ NMR can serve as a strong evidence for face to face complexation of the probed molecule with aromatic structures. In previous studies, the observed ${ }^{1} \mathrm{H} /{ }^{13} \mathrm{C}$ NMR upfield chemical shifts have been used to support $\pi-\pi$ EDA complexation between $\pi$-electronacceptor compounds and PAHs ( $\pi$-electron-donors) [32]. In this study, prominent ${ }^{1} \mathrm{HNM}$ upfield shifts are observed for $\mathrm{Nor}^{+}$in mixtures with PAHs ( $\pi$-electron-donors). For example, the bands of $-\mathrm{H}$ on amino group and methylene on $-\mathrm{CH}_{2} \mathrm{CH}_{3}$ group of $\mathrm{Nor}^{+}$ were shifted up to $0.3 \mathrm{ppm}$ and $0.25 \mathrm{ppm}$ in the presence of PYR, respectively (Fig. 4). The magnitudes of upfield shifts increased with an increase of PAHs concentrations and also correlated well with $\pi$-donor strength of PAHs (PYR $>$ PHEN $>$ NAPH). The observed trends clearly demonstrated formation of $\pi-\pi$ EDA interaction and cation $-\pi$ bonding between $\mathrm{Nor}^{+}$and PAHs in solution.

\subsection{Solubility enhancement studies}

To further verify the cation- $\pi$ interaction between positively charged heterocyclic $\mathrm{N}$ atom of $\mathrm{Nor}^{+}$and PAHs, a solubility enhancement experiment was conducted. Fig. 5 shows the enhanced solubility of NAPH, PHEN, PYR vs. concentrations of Nor in aqueous solution at $\mathrm{pH} 4.0$ and 9.0, respectively. Clearly, the presence of Nor increases the solubility of three PAHs, and the solubility enhancement correlates with $\pi$-donor ability of PAH (PYR > PHEN > NAPH). For example, at pH 4.0, the solubility of NAPH, PHEN and PYR increased up to 1.5 times, 2.8 times, and 17.7 times, respectively, in the presence of Nor compared to that in the absence of Nor due to possible occurrence of $\pi-\pi$, and cation $-\pi$ interaction 


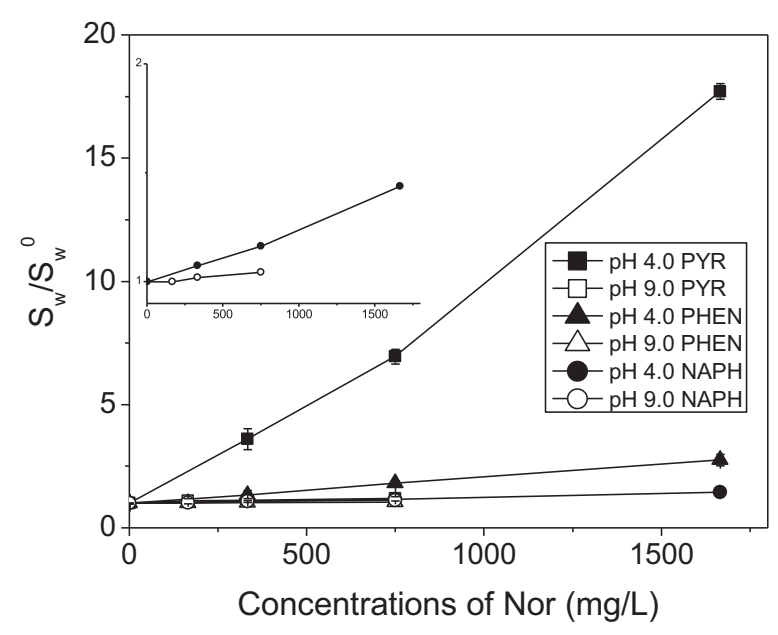

Fig. 5. Ratio of saturated solubility of NAPH, PHEN, and PYR in the presence of Nor to that in the absence of Nor vs. Nor concentrations at $\mathrm{pH} 4.0$ and 9.0. The insert represents the solubility ratio of NAPH in the presence and absence of Nor.

at the examined maximum concentration of Nor $^{+}$. In contrast, $\mathrm{Nor}^{-}$ only increased the solubility of three PAHs slightly at pH 9.0 (up to 1.1 times for NAPH, 1.1 times for PHEN, and 1.2 times for PYR). These results of the maximum solubility enhancement by $\mathrm{Nor}^{+}$for PYR (17.7 times) than that by Nor $^{-}$(1.2 times) strongly suggested that PAHs form cation $-\pi$ complexes with $\mathrm{Nor}^{+}$in aqueous solution.

\section{Conclusions}

In view of the foregoing study, it was found that Nor had different effects on different organic contaminants. For 1,3-DNB, the presence of Nor suppressed its sorption on $\mathrm{K}^{+}$-montmorillonite due to their competition for the same sorption sites. FTIR study reveals that 1,3-DNB was sorbed on $\mathrm{K}^{+}$-montmorillonite via cation-polar interaction of its $-\mathrm{NO}_{2}$ groups with exchangeable $\mathrm{K}^{+}$and $\mathrm{n}-\pi$ electron donor-acceptor (EDA) interaction of 1,3-DNB ( $\mathrm{e}^{-}$acceptors) with oxygens of montmorillonite siloxane surfaces ( $e^{-}$donors). While Nor was also sorbed on these sites through cation exchange, cation bridging and/or surface complexation.

In contrast, Nor increased the sorption of three PAHs on $\mathrm{K}^{+}$montmorillonite and the enhanced magnitude was positively correlated with $\pi$-donor strength of three PAHs. The enhanced effect of Nor on PAHs sorption was due to $\pi-\pi$ interaction between the electron-depleted quinoline ring ( $\pi$-electron acceptor) of Nor and PAHs ( $\pi$-electron donor). Different species of Nor had different effects on the sorption of PAHs. Compared to $\mathrm{Nor}^{+}$and Nor ${ }^{-}$, Nor $^{ \pm}$increased the sorption of PHEN and PYR more pronounced, which was attributed to additional formation of cation- $\pi$ interaction between positively charged heterocyclic $\mathrm{N}$ atom of sorbed Nor $^{ \pm}$and PAHs.

\section{Acknowledgements}

This work was funded by the National Natural Science Foundation of China (grant numbers: 41071308, 40730740 and 20807052) and the Youth Fund of State Key Laboratory of Environmental Chemistry and Ecotoxicology QN2009-07.

\section{Appendix A. Supplementary data}

Supplementary data associated with this article can be found, in the online version, at doi:10.1016/j.jhazmat.2011.11.087.

\section{References}

[1] E.M. Golet, A. Strehler, A.C. Alder, W. Giger, Determination of fluoroquinolone antibacterial agents in sewage sludge and sludge-treated soil using accelerated solvent extraction followed by solid-phase extraction, Anal. Chem. 74 (2002) 5455-5462.

[2] A. Nowara, J. Burhenne, M. Spiteller, Binding of fluoroquinolone carboxylic acid derivatives to clay minerals, J. Agric. Food Chem. 45 (1997) 459-1463.

[3] C. Gu, K.G. Karthikeyan, Sorption of the antimicrobial ciprofloxacin to aluminum and iron hydrous oxides, Environ. Sci. Technol. 39 (2005) 9166-9173.

[4] P. Trivedi, D. Vasudevan, Spectroscopic investigation of ciprofloxacin speciation at the goethite-water interface, Environ. Sci. Technol. 41 (2007) 3153-3158.

[5] A.J. Carrasquillo, G.L. Bruland, A.A. Mackay, D. Vasudevan, Sorption of ciprofloxacin and oxytetracycline zwitterions to soils and soil minerals: influence of compound structure, Environ. Sci. Technol. 42 (2008) 7634-7642.

[6] D. Vasudevan, G.L. Bruland, B.S. Torrance, V.G. Upchurch, A.A. MacKay, pHdependent ciprofloxacin sorption to soils: interaction mechanisms and soil factors influencing sorption, Geoderm 151 (2009) 68-76.

[7] Z.Y. Wang, X.D. Yu, B. Pan, B.S. Xing, Norfloxacin sorption and its thermodynamics on surface-modified carbon nanotubes, Environ. Sci. Technol. 44 (2010) 978-984.

[8] K.W. Weissmahr, M. Hildenbrand, R.P. Schwarzenbach, S.B. Haderlein, Laboratory and field scale evaluation of geochemical controls on groundwater transport of nitroaromatic ammunition residues, Environ. Sci. Technol. 33 (1999) 2593-2600.

[9] D.R. Wellington, W.R. Mitchell, In vitro cytotoxicity of certain munition nitroaromatic compounds, Chemosphere 23 (1991) 363-373.

[10] S.B. Haderlein, K.W. Weissmahr, R.P. Schwarzenbach, Specific adsorption of nitroaromatic explosives and pesticides to clay minerals, Environ. Sci. Technol. 30 (1996) 612-622.

[11] K.W.Weissmahr, S.B. Haderlein, R.P. Schwarzenbach, R. Hany, R. Nuesch, In situ spectroscopic investigations of adsorption mechanisms of nitroaromatic compounds at clay minerals, Environ. Sci. Technol. 31 (1997) 240-247.

[12] S.A. Boyd, G. Sheng, B.J. Teppen, C.T. Johnston, Mechanisms for the adsorption of substituted nitrobenzenes by smectite clays, Environ. Sci. Technol. 35 (2001) 4227-4234.

[13] C.T. Johnston, M.F. De Oliveira, B.J. Teppen, G. Sheng, S.A. Boyd, Spectroscopic study of nitroaromatic-smectite sorption mechanisms, Environ. Sci. Technol. 35 (2001) 4767-4772.

[14] H. Li, B.J. Teppen, C.T. Johnston, S.A. Boyd, Thermodynamics of nitroaromatic compound adsorption from water by smectite clay, Environ. Sci. Technol. 38 (2004) 5433-5442.

[15] X.L. Qu, Y.J. Zhang, H. Li, S.R. Zheng, D.Q. Zhu, Probing the specific sorption sites on montmorillonite using nitroaromatic compounds and hexafluorobenzene, Environ. Sci. Technol. 45 (2011) 2209-2216.

[16] G. Cornelissen, Ö. Gustafsson, T.D. Bucheli, M.T.O. Jonker, A.A. Koelmans, P.C.M. Van Noort, Extensive sorption of organic compounds to black carbon, coal, and kerogen in sediments and soils: mechanisms and consequences for distribution, bioaccumulation, and biodegradation, Environ. Sci. Technol. 39 (2005) 6881-6895.

[17] L.S. Hundal, M.L. Thompson, D.A. Laird, A.M. Carmo, Sorption of phenanthrene by reference smectites, Environ. Sci. Technol. 35 (2001) 3456-3461.

[18] C. Tanford, The Hydrophobic Effect: Formation of Micelles and Biological Membranes, 2nd ed., John Wiley \& Sons, New York, 1980.

[19] K.U. Goss, S.J. Eisenreich, Adsorption of organic compounds from water to mineral surfaces: a theoretical approach, In Preprints of papers, Division of Environmental Chemistry. Am. Chem. Soc., Washington, DC, 35 (1995) 540-543.

[20] C.T. Chiou, T.D. Shoup, P.E. Porter, Mechanistic roles of soil humus and minerals in the sorption of nonionic organic compounds from aqueous and organic solutions, Org. Geochem. 8 (1985) 9-14.

[21] Y.H. Su, Y.G. Zhu, G.Y. Sheng, C.T. Chiou, Linear adsorption of nonionic organic compounds from water onto hydrophilic minerals: silica and alumina, Environ. Sci. Technol. 40 (2006) 6949-6954.

[22] D.Q. Zhu, B.E. Herbert, M.A. Schlautman, E.R. Carraway, J. Hur, Cation- $\pi$ bonding: a new perspective on the sorption of polycyclic aromatic hydrocarbons to mineral surfaces, J. Environ. Qual. 33 (2004) 1322-1330.

[23] W. Chen, L. Duan, L.L. Wang, D.Q. Zhu, Adsorption of hydroxyl- and aminosubstituted aromatics to carbon nanotubes, Environ. Sci. Technol. 42 (2008) 6862-6868.

[24] K. Yang, W.H. Wu, Q.F. Jing, W. Jiang, B.S. Xing, Competitive adsorption of naphthalene with 2, 4-dichlorophenol and 4-chloroaniline on multiwalled carbon nanotubes, Environ. Sci. Technol. 44 (2010) 3021-3027.

[25] X.L. Qu, P. Liu, D.Q. Zhu, Enhanced sorption of polycyclic aromatic hydrocarbons to tetra-alkyl ammonium modified smectites via cation- $\pi$ interactions, Environ. Sci. Technol. 42 (2008) 1109-1116.

[26] X.L. Wang, S. Tao, B.S. Xing, Sorption and competition of aromatic compounds and humic acid on multiwalled carbon nanotubes, Environ. Sci. Technol. 43 (2009) 6214-6219.

[27] M.E. Sumner, W.P. Miller, Cation exchange capacity and exchange coefficients, in: D.L. Sparks (Ed.), Methods of Soil Analysis: Part 3. Chemical Methods, American Society of Agronomy-Soil Science Society of America, Madison, WI, 1996, pp. 1201-1229.

[28] R.A. Figueroa, A. Leonard, A.A. Mackay, Modeling tetracycline antibiotic sorption to clays, Environ. Sci. Technol. 38 (2004) 476-483. 
[29] J. Ha, T.P. Trainor, F. Farges, G.E. Brown, Interaction of Zn(II) with hematite nanoparticles and microparticles: Part 2. ATR-FTIR and EXAFS study of the aqueous $\mathrm{Zn}(\mathrm{II}) /$ Oxalate/hematite ternary system, Langmuir 25 (2009) 5586-5593.

[30] M. Keiluweit, M. Kleber, Molecular-level interactions in soils and sediments: the role of aromatic $\pi$-systems, Environ. Sci. Technol. 43 (2009) 3421-3429.
[31] R.T. Yang, A.J. Hernández-Maldonado, F.H. Yang Desulfurization of transportation fuels with zeolites under ambient conditions, Science 301 (2003) 79-81.

[32] L.L. Ji, W.Chen, L. Duan, D.Q. Zhu, Mechanisms for strong adsorption of tetracycline to carbon nanotubes: a comparative study using activated carbon and graphite as adsorbents, Environ. Sci. Technol. 43 (2009) 2322-2327. 